\title{
Effect of Troponin I Elevation on Duration of Mechanical Ventilation and Length of Intensive Care Unit Stay in Patients With Sepsis
}

\author{
Mohammed Abdalla $^{\text {a, c }}$, Sumit Sohal ${ }^{\mathrm{a}}$, Baha'a Al-Azzam ${ }^{\mathrm{a}}$, Wiam Mohamed $^{\mathrm{b}}$
}

\begin{abstract}
Background: Sepsis is a leading cause of mortality and morbidity. Recent studies suggest that troponin elevation is associated with increased mortality in sepsis patients. The purpose of this study is to determine the effect of troponin elevation on mechanical ventilation duration and intensive care unit (ICU) length of stay in patients with sepsis. Additionally, we investigated the association between troponin elevation and septic shock.
\end{abstract}

Methods: The study is a retrospective observational cohort study, conducted in a community teaching hospital between October 2015 and April 2018. All adult ICU patients with sepsis who required invasive mechanical ventilation were included. Primary outcomes were mechanical ventilation duration and ICU length of stay. Secondary outcomes were hospital length of stay, in-hospital mortality and association with septic shock.

Results: A total of 125 patients were included. Troponin was elevated in $36 \%(45 / 125)$ of the patients. Duration of mechanical ventilation in hours was longer for troponin-positive group (troponin positive: 55.6 $\mathrm{h}$ vs. troponin negative: $49.6 \mathrm{~h}, 95 \% \mathrm{CI} 0.001-0.063, \mathrm{P}=0.032$ ). ICU length of stay was similar (troponin positive: 4.6 days vs. troponin negative: 4.3 days, $95 \%$ CI $0.130-0.270$ ). Septic shock had higher occurrence in elevated troponin group (troponin positive: $62.2 \%$ $(28 / 45)$ vs. troponin negative: $43.8 \%(35 / 80)$, risk ratio (RR) $1.4, \mathrm{P}=$ $0.04,95 \%$ CI 1.015 - 1.99).

Conclusions: Troponin elevation was associated with longer duration of mechanical ventilation in patients admitted to ICU with sepsis. Troponin elevation was associated with higher risk for development of septic shock. No significant effect was observed in ICU length of stay and hospital length of stay.

Keywords: Sepsis; Troponin; Intensive care unit; Critical care; Septic shock; Mortality; Length of stay

Manuscript submitted December 8, 2018, accepted December 26, 2018

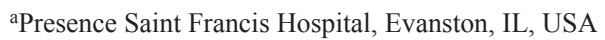

bUniversity of Khartoum, Khartoum, Sudan

${ }^{\mathrm{c} C o r r e s p o n d i n g ~ A u t h o r: ~ M o h a m m e d ~ A b d a l l a, ~ P r e s e n c e ~ S a i n t ~ F r a n c i s ~ H o s p i-~}$ tal, Evanston, IL 60202, USA. Email: ma7amad_3omar@yahoo.com

doi: https://doi.org/10.14740/jocmr3713

\section{Introduction}

Sepsis is a leading cause of mortality and morbidity in the USA. More than 1.5 million patients in the USA are diagnosed with sepsis annually. Of them, about 250,000 die from sepsis and one in three patients who die in a hospital have sepsis [1]. Troponin elevation in patients with sepsis is a predictor of a poor prognosis and of mortality [2].

One of the characteristics of sepsis, and part of the definition of the systemic inflammatory response syndrome (SIRS), is an increase in respiratory rate [3] which increases the work of breathing and affects blood flow to the diaphragm [4], which further increases cardiac demands.

This study was planned to determine the effect of troponin elevation on invasive mechanical ventilation duration and length of stay of sepsis patients in the intensive care unit (ICU). We hypothesized that lung injury in sepsis requiring mechanical ventilation results in increased work of breathing which increases cardiac demand leading to ischemia reflected as troponin elevation. This can help in prognosis, treatment decisions and discussions with family regarding further treatment plans and goals of care.

\section{Materials and Methods}

\section{Design, setting and patient selection}

The design of the study is a retrospective observational cohort study. It was done in Presence Saint Francis Hospital, a community teaching hospital in Evanston, IL with 16 ICU beds and 10 progressive care unit beds. The study was conducted between October 2015 and April 2018. The institutional review board of the hospital approved the study protocol (SFH\#00347). As the study was retrospective the board waived obtaining informed consent.

All adult patients admitted to ICU of Presence Saint Francis Hospital within the period of October 2015 to April 2018 with diagnosis of sepsis who required intubation and mechanical ventilation were included in the study.

Electronic medical records were reviewed for clinical findings, investigations and the course of hospitalization. We used ICD-10 codes for sepsis and mechanical ventilation combined with clinical judgment to identify patients for the study. 
Sepsis was defined as two or more SIRS criteria with possible or confirmed source of infection. Troponin I elevation was defined as elevation of serum or plasma troponin I above the 99th percentile of the upper reference limit for the assay used.

Patients who had been diagnosed with acute coronary syndrome (ACS) or had evidence of ACS on echocardiogram or heart catheterization were excluded from the study.

\section{Data collection}

Data were gathered for analysis, specifically age, gender, vital signs prior to ICU admission, confusion prior to ICU admission (defined as Glasgow coma scale less than 15), SIRS prior to ICU admission, occurrence of septic shock during the admission, lactate serum level and white blood cell (WBC) count on admission, troponin I positivity as defined above, duration of mechanical ventilation, duration of ICU length of stay, hospital length of stay, mortality during the admission, and source of infection and comorbidities.

We described sources of infection as respiratory, urinary tract, intra-abdominal, cutaneous and others. Comorbidities included congestive heart failure, coronary artery disease, diabetes mellitus, chronic obstructive pulmonary disease and chronic kidney disease.

\section{End points}

Primary outcomes were mechanical ventilation duration in hours and ICU length of stay (days), and secondary outcomes were hospital length of stay (days), in-hospital mortality and occurrence of troponin elevation with septic shock.

In-hospital mortality was defined as death occurring during the index admission. Septic shock was defined as sepsis requiring vasopressors to maintain a mean arterial pressure (MAP) of $\geq 65 \mathrm{~mm} \mathrm{Hg}$ despite adequate fluid resuscitation.

\section{Statistical analysis}

SPSS software (IBM Corp. Released 2017. IBM SPSS Statistics for Windows, Version 25.0. Armonk, NY, USA) was used for statistical analysis, continuous data were compared using Mann-Whitney U test, and categorical data were compared using Chi-square test. Risk ratio (RR) and 95\% confidence interval $(95 \% \mathrm{CI})$ were calculated, and $\mathrm{P}$ values less than 0.05 were considered statistically significant.

\section{Results}

The patients' flow diagram is shown in Figure 1. Total number of patients who were admitted to ICU with sepsis and underwent intubation and mechanical ventilation was 320 . Troponin was checked in 139 patients. Fourteen patients were excluded as they were diagnosed with ACS. The number of patients in- cluded in the study was 125 .

Females patients were $46(36.8 \%)$, and male patients were $79(63.2 \%)$. Troponin was positive in 45 patients $(36 \%)$, and negative in 80 patients $(64 \%)$. Characteristics of patients are presented in Table 1.

The most common source of infection was respiratory in 100 patients $(80 \%)$, and other sources of infection are demonstrated in Table 2.

Septic shock developed in 63 patients during the admission $(50.4 \%)$. Twenty-three patients died during the admission (18.4\%). Code blue was called for five patients (4\%).

As demonstrated in Table 3, septic shock developed in 28 patients in troponin-positive group $(28 / 45,62.2 \%)$, and in 35 patients in troponin-negative group $(35 / 80,43.8 \%)(\mathrm{P}=0.04$, RR $1.4,95 \%$ CI 1.015 - 1.99). The results were statistically significant. Mortality during the admission was $24.4 \%(11 / 45)$ in troponin-positive, and $15 \%(12 / 80)$ in troponin-negative group $(\mathrm{P}=0.19$, RR 1.6, 95\% CI $0.78-3.4)$. The results were not statistically significant.

The average duration of mechanical ventilation in hours for troponin-positive group was $55.6 \mathrm{~h}$, and $49.6 \mathrm{~h}$ in troponinnegative patients $(\mathrm{P}=0.032,95 \%$ CI $0.001-0.063)$. The results were statistically significant. The average ICU length of stay was 4.6 days in troponin-positive group, and 4.3 days in troponin negative patients $(95 \%$ CI $0.130-0.270)$. The average hospital length of stay was 9.7 days in troponin-positive group, and 8.4 days in troponin-negative patients $(95 \% \mathrm{CI}$ $0.187-0.341$ ), as shown in Table 4.

\section{Discussion}

Sepsis is caused by the inefficiency of control mechanisms and infection containment. It is characterized by an inflammatory reaction to infection and manifestations of organ dysfunction resulting from alterations in the microcirculation [5]. During sepsis, regional blood flows abnormally, leading to microcirculation disturbance causing ischemia of various organs, including the heart $[6,7]$ and troponin is a sensitive marker of myocardial injury and "non-coronary troponin" elevation is common among critically ill patients [8].

Our study investigated relationship of this myocardial injury in intubated patients with sepsis which was reflected by a positive troponin level to duration of mechanical ventilation in patients admitted to ICU. In our study $36 \%$ of the patients had elevated troponin. Lim et al demonstrated that elevated troponin levels are observed in $43 \%$ across all intensive care patient groups (interquartile range 21-59\%) [9], whereas Bessiere et al described the prevalence of positive troponin in patients admitted with sepsis to be around 61\% [10] which was similar to the study by Sheyin et al $(60.5 \%)$ [2]. The percentage in our study is lower, but not every patient who was admitted with sepsis had their troponin checked in our study.

Prior studies on sepsis and septic shock presented conflicting data on the association of clinical outcomes with troponin elevation. Various studies including those by Vallabhajosyula et al [11], Yang et al [12], Vasile et al [13], de Groot et al [14], Sheyin et al [2], John et al [15] and Bessiere et al [10] have 


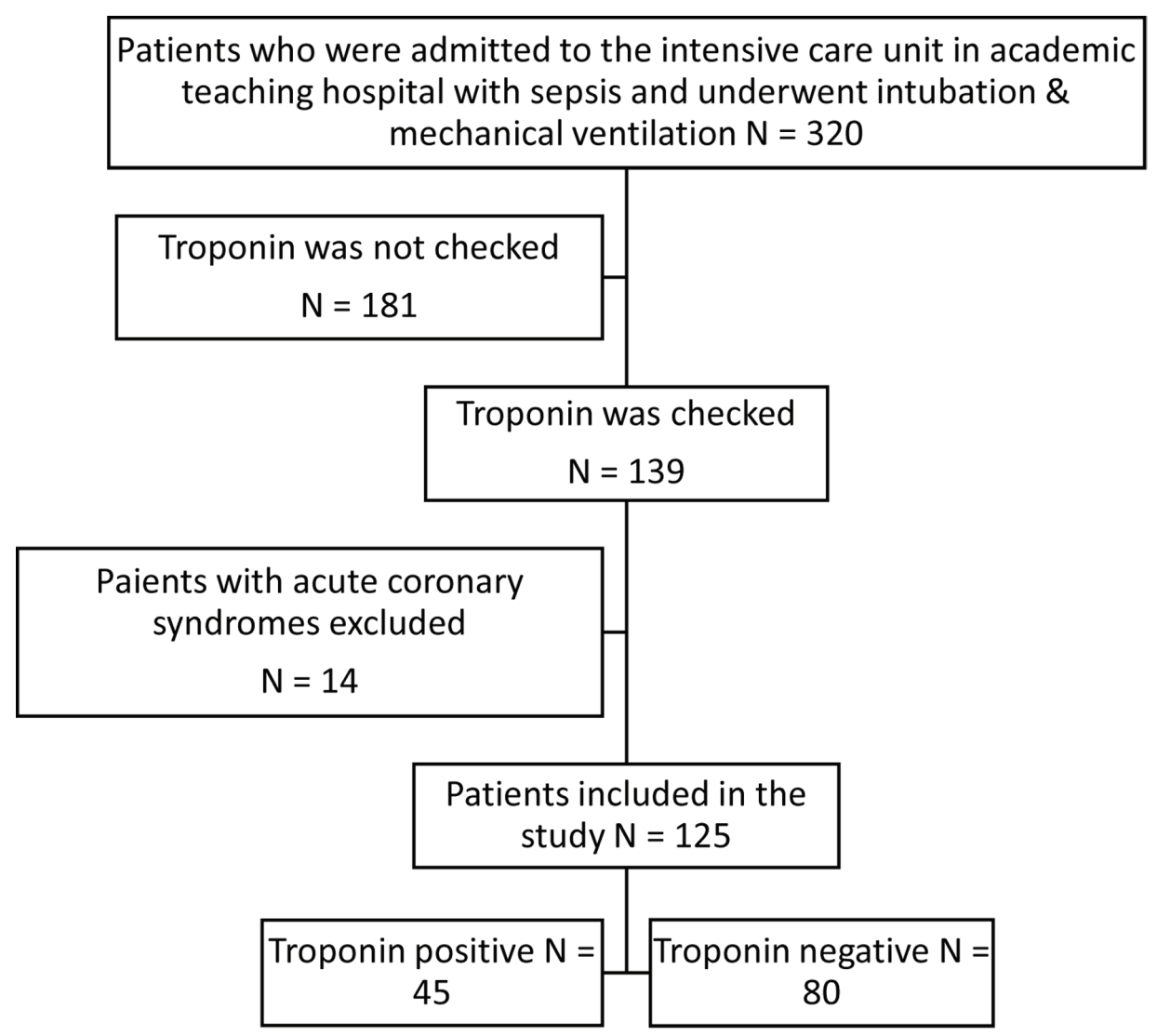

Figure 1. Flow chart of patients who were admitted to ICU with possible sepsis and underwent intubation and mechanical ventilation.

concluded that troponin elevation was associated with adverse outcomes and increased mortality in patents admitted with sepsis. Also Rosjo et al stated that troponin elevation is associated with survival in sepsis and may have a role as an early marker of shock [16]. However some other studies such as Tiruvoipati et al [17], Mehta et al [18] and Landesberg et al [19] refuted this claim. In our study we found troponin elevation to be a predictor of development of septic shock. Our study showed mortality was higher in patients with elevated troponin, but our results did not reach statistical significance $(\mathrm{P}=0.19$, RR 1.6, 95\% CI 0.78 - 3.4).

Patients who were admitted with sepsis and had an elevated troponin level on admission were at risk of developing septic shock during the course of their stay $(\mathrm{P}=0.04, \mathrm{RR} 1.4$, $95 \%$ CI 1.015 - 1.99). This indicates that troponin can be used as an early marker for development of shock in the patients admitted with sepsis.

Studies such as Lim et al [9] identified that patients with elevated troponin levels had increased hospital length of stay, while studies as Tiruvoipati et al [17] did not shown an association. Our study did not show increased length of stay in the hospital in patients with elevated troponin and the results were statistically significant (95\% CI $0.187-0.341)$.

The heart and lungs interact during each ventilation cycle. The application of mechanical ventilation and subsequent removal change normal ventilatory mechanics and produce alterations in cardiac preload and afterload that influence global hemodynamic state and delivery of oxygen and nutrients [20]. Guest et al in their study demonstrated that patients with cardiac injury were more frequently in need of mechanical ventilation as compared to other critically ill patient, which was re-demonstrated by Blamoun et al $[21,22]$. Our study demonstrated that the average duration of mechanical ventilation in hours to be longer in patients with elevated troponin than in other patients (55.6 h vs. 49.6 h, $\mathrm{P}=0.032,95 \%$ CI $0.001-0.063$ ).

Studies including ours showed the association of troponin with increased adverse outcomes which may indicate that early aggressive resuscitation can improve myocardial injury and survival [23-26]. The early identification of this subset of septic patients can help identifying patients at risk of developing septic shock and may help in making clinical decisions on early goal-directed therapy.

\section{Conclusions}

Troponin I elevation is associated with longer duration of mechanical ventilation in patients admitted to ICU with sepsis. Troponin I elevation is associated with higher risk for development of septic shock. No significant effect was observed on 
Table 1. Characteristics of Patients Who Were Admitted to ICU With Possible Sepsis and Underwent Intubation and Mechanical Ventilation

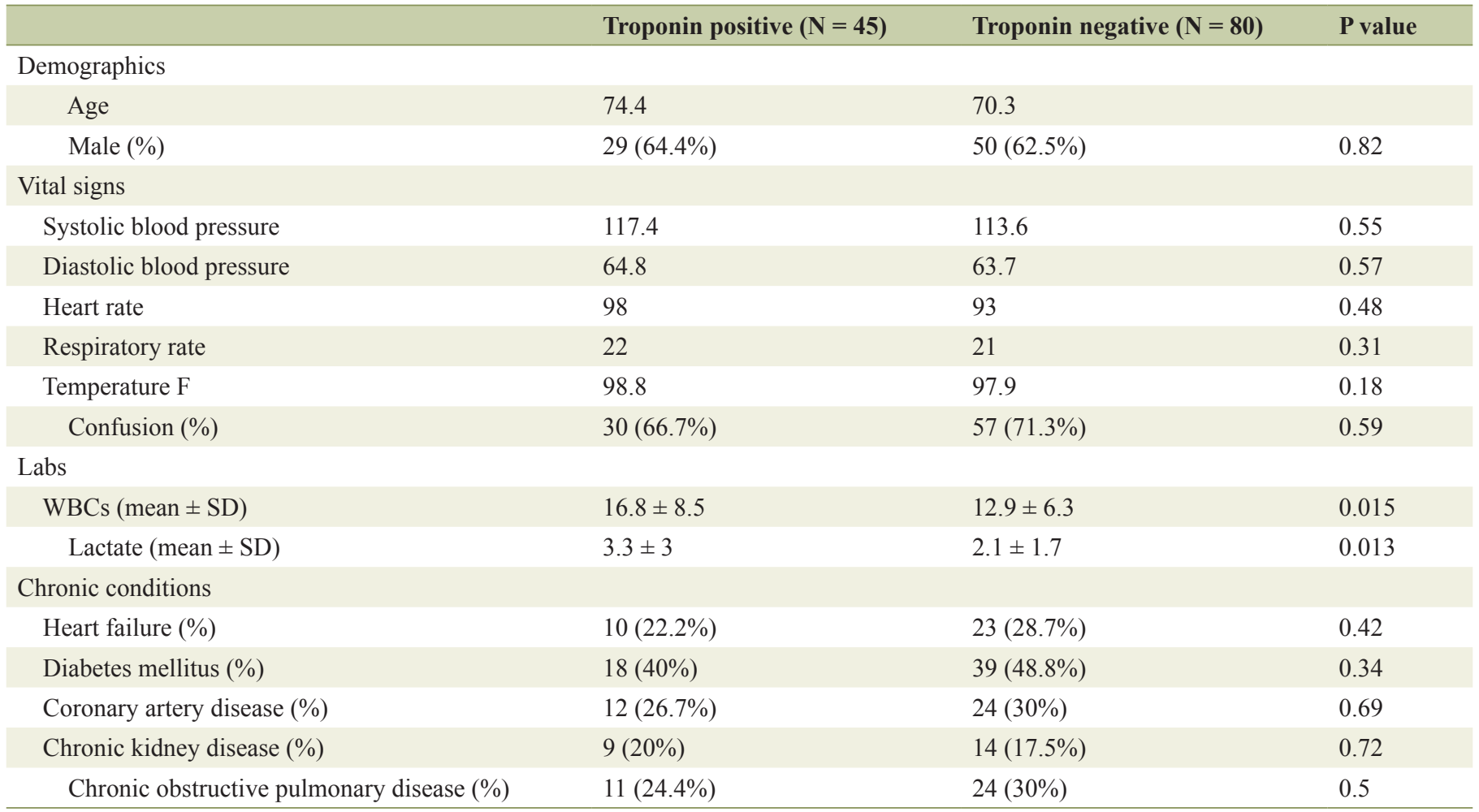

Table 2. Sources of Infection in Patients Who Were Admitted to ICU With Possible Sepsis and Underwent Intubation and Mechanical Ventilation

\begin{tabular}{llll}
\hline & Troponin negative $(\mathbf{N}=\mathbf{8 0})$ & Troponin positive $(\mathbf{N}=45)$ & \\
\hline Respiratory & $62(77.5 \%)$ & $38(84.4 \%)$ & $100(80 \%)$ \\
Urinary tract & $12(15 \%)$ & $4(8.9 \%)$ & $16(12.8 \%)$ \\
Intraabdominal & $5(6.3 \%)$ & $2(4.4 \%)$ & $7(5.6 \%)$ \\
Cutaneous & $1(1.3 \%)$ & $0(0 \%)$ & $1(0.8 \%)$ \\
Others & $0(0 \%)$ & $1(2.2 \%)$ & $1(0.8 \%)$ \\
\hline
\end{tabular}

Table 3. Septic Shock and In-Hospital Mortality in Intubated ICU Patients Admitted With Sepsis

\begin{tabular}{lllll}
\hline & Troponin positive $(\mathbf{N}=\mathbf{4 5})$ & Troponin negative $(\mathbf{N}=\mathbf{8 0})$ & RR $(\mathbf{9 5 \%}$ CI) & P value \\
\hline Septic shock (\%) & $28(62.2 \%)$ & $35(43.8 \%)$ & $1.42(1.015-1.99)$ & 0.04 \\
In-hospital mortality (\%) & $11(24.4 \%)$ & $12(15 \%)$ & $1.6(0.78-3.4)$ & 0.19
\end{tabular}

Table 4. Duration of Mechanical Ventilation (hours), ICU Length of Stay (days) and Hospital Length of Stay (days) in Intubated ICU Patients Admitted With Sepsis

\begin{tabular}{|c|c|c|c|c|}
\hline & $\begin{array}{l}\text { Troponin positive } \\
(\mathrm{N}=45)\end{array}$ & $\begin{array}{l}\text { Troponin negative } \\
(\mathrm{N}=\mathbf{8 0})\end{array}$ & $95 \% \mathrm{CI}$ & P value \\
\hline Duration of mechanical ventilation in hours (mean $\pm \mathrm{SD}$ ) & $55.6 \pm 24.8$ & $49.6 \pm 33$ & $0.001-0.063$ & 0.032 \\
\hline Hospital length of stay in days (mean $\pm \mathrm{SD}$ ) & $9.7 \pm 6$ & $8.4 \pm 4.4$ & $0.187-0.341$ & 0.264 \\
\hline
\end{tabular}


duration of ICU length of stay and hospital length of stay.

\section{Consent for Publication}

Not applicable.

\section{Conflict of Interest}

The authors declare that they have no conflict of interest.

\section{Data Availability}

Data will be available upon reasonable request with permission from the institution.

\section{Author Contributions}

MA designed the study, collected and analyzed data, and wrote the first draft of the manuscript. SS collected data and participated in writing and revision of the manuscript. BA extracted data and revised manuscript. WM participated in the analysis of the data and revised the manuscript. All authors read and approved the final manuscript.

\section{Ethics Approval and Consent to Participate}

The institutional review board of Presence Saint Francis Hospital approved the study protocol (SFH\#00347). As the study was retrospective the board waived obtaining the informed consent.

\section{Financial Support}

No source of funding was received for this research.

\section{References}

1. https://www.cdc.gov/sepsis/datareports/index.html.

2. Sheyin O, Davies O, Duan W, Perez X. The prognostic significance of troponin elevation in patients with sepsis: a meta-analysis. Heart Lung. 2015;44(1):75-81.

3. Bone RC, Fisher CJ, Jr., Clemmer TP, Slotman GJ, Metz CA, Balk RA. Sepsis syndrome: a valid clinical entity. Methylprednisolone Severe Sepsis Study Group. Crit Care Med. 1989;17(5):389-393.

4. Buchler B, Magder S, Roussos C. Effects of contraction frequency and duty cycle on diaphragmatic blood flow. J Appl Physiol (1985). 1985;58(1):265-273.

5. Clemente G, Tuttolomondo A, Colomba D, Pecoraro R, Renda C, Della Corte V, Maida C, et al. When sepsis affects the heart: A case report and literature review.
World J Clin Cases. 2015;3(8):743-750.

6. Jozwiak M, Persichini R, Monnet X, Teboul JL. Management of myocardial dysfunction in severe sepsis. Semin Respir Crit Care Med. 2011;32(2):206-214.

7. Wang J, Ji W, Xu Z, Pan T. Clinical significance of plasma levels of brain natriuretic peptide and cardiac troponin $\mathrm{T}$ in patients with sepsis. Exp Ther Med. 2016;11(1):154156.

8. Zochios V, Valchanov K. Raised cardiac troponin in intensive care patients with sepsis, in the absence of angiographically documented coronary artery disease: A systematic review. J Intensive Care Soc. 2015;16(1):52-57.

9. Lim W, Qushmaq I, Devereaux PJ, Heels-Ansdell D, Lauzier F, Ismaila AS, Crowther MA, et al. Elevated cardiac troponin measurements in critically ill patients. Arch Intern Med. 2006;166(22):2446-2454.

10. Bessiere F, Khenifer S, Dubourg J, Durieu I, Lega JC. Prognostic value of troponins in sepsis: a meta-analysis. Intensive Care Med. 2013;39(7):1181-1189.

11. Vallabhajosyula S, Sakhuja A, Geske JB, Kumar M, Poterucha JT, Kashyap R, Kashani K, et al. Role of admission troponin- $\mathrm{T}$ and serial troponin- $\mathrm{T}$ testing in predicting outcomes in severe sepsis and septic shock. J Am Heart Assoc. 2017;6(9):e005930.

12. Yang Z, Qdaisat A, Hu Z, Wagar EA, Reyes-Gibby C, Meng QH, Yeung SC. Cardiac troponin is a predictor of septic shock mortality in cancer patients in an emergency department: a retrospective cohort study. PLoS One. 2016;11(4):e0153492.

13. Vasile VC, Chai HS, Abdeldayem D, Afessa B, Jaffe AS. Elevated cardiac troponin T levels in critically ill patients with sepsis. Am J Med. 2013;126(12):1114-1121.

14. de Groot B, Verdoorn RC, Lameijer J, van der Velden J. High-sensitivity cardiac troponin $\mathrm{T}$ is an independent predictor of inhospital mortality in emergency department patients with suspected infection: a prospective observational derivation study. Emerg Med J. 2014;31(11):882888.

15. John J, Woodward DB, Wang Y, Yan SB, Fisher D, Kinasewitz GT, Heiselman D. Troponin-I as a prognosticator of mortality in severe sepsis patients. J Crit Care. 2010;25(2):270-275.

16. Rosjo H, Varpula M, Hagve TA, Karlsson S, Ruokonen E, Pettila V, Omland T, et al. Circulating high sensitivity troponin $\mathrm{T}$ in severe sepsis and septic shock: distribution, associated factors, and relation to outcome. Intensive Care Med. 2011;37(1):77-85.

17. Tiruvoipati R, Sultana N, Lewis D. Cardiac troponin I does not independently predict mortality in critically ill patients with severe sepsis. Emerg Med Australas. 2012;24(2):151-158.

18. Mehta S, Granton J, Gordon AC, Cook DJ, Lapinsky S, Newton G, Bandayrel K, et al. Cardiac ischemia in patients with septic shock randomized to vasopressin or norepinephrine. Crit Care. 2013;17(3):R117.

19. Landesberg G, Jaffe AS, Gilon D, Levin PD, Goodman S, Abu-Baih A, Beeri R, et al. Troponin elevation in severe sepsis and septic shock: the role of left ventricular diastolic dysfunction and right ventricular dilatation*. Crit 
Care Med. 2014;42(4):790-800.

20. Frazier SK. Cardiovascular effects of mechanical ventilation and weaning. Nurs Clin North Am. 2008;43(1):1-15; V.

21. Guest TM, Ramanathan AV, Tuteur PG, Schechtman KB, Ladenson JH, Jaffe AS. Myocardial injury in critically ill patients. A frequently unrecognized complication. JAMA. 1995;273(24):1945-1949.

22. Blamoun AI, Batty GN, Rashid A, Sheikh M, DeBari VA, Khan MA. Cardiac troponin levels and mechanical ventilator dependence in patients with septic shock. Chest. 2007;132(4):554A.

23. Zhang XL, Qin YJ, Bian XH, Dong SM. [Effects of fluid resuscitation on myocardial injury in patients with severe sepsis and septic shock]. Zhongguo Wei Zhong Bing Ji Jiu Yi Xue. 2012;24(4):222-224.

24. Turner A, Tsamitros M, Bellomo R. Myocardial cell injury in septic shock. Crit Care Med. 1999;27(9):1775-1780.

25. Chagnon F, Bentourkia M, Lecomte R, Lessard M, Lesur O. Endotoxin-induced heart dysfunction in rats: assessment of myocardial perfusion and permeability and the role of fluid resuscitation. Crit Care Med. 2006;34(1):127133.

26. Hussain N. Elevated cardiac troponins in setting of systemic inflammatory response syndrome, sepsis, and septic shock. ISRN Cardiol. 2013;2013:723435. 